\title{
A $q$-HANKEL TRANSFORM ASSOCIATED TO THE QUANTUM LINKING GROUPOID FOR THE QUANTUM $S U(2)$ AND $E(2)$ GROUPS
}

\author{
KENNY DE COMMER AND ERIK KOELINK \\ (Communicated by Walter Van Assche)
}

\begin{abstract}
A q-analogue of Erdélyi's formula for the Hankel transform of the product of Laguerre polynomials is derived using the quantum linking groupoid between the quantum $S U(2)$ and $E(2)$ groups. The kernel of the $q$-Hankel transform is given by the $1 \varphi_{1}-q$-Bessel function, and then the transform of a product of two Wall polynomials times a $q$-exponential is calculated as a product of two Wall polynomials times a $q$-exponential.
\end{abstract}

\section{INTRODUCTION}

In 1938 Arthur Erdélyi [3] proved the following formula for the Hankel transform of the product of two Laguerre polynomials:

$$
\begin{gathered}
\int_{0}^{\infty} x^{\nu} e^{-x^{2}} L_{m}^{(\nu-\sigma)}\left(x^{2}\right) L_{n}^{(\sigma)}\left(x^{2}\right) J_{\nu}(2 x y) x d x= \\
\frac{(-1)^{m+n}}{2} y^{\nu} e^{-y^{2}} L_{m}^{(\sigma-m+n)}\left(y^{2}\right) L_{n}^{(\nu-\sigma+m-n)}\left(y^{2}\right)
\end{gathered}
$$

using integral representations for the Laguerre polynomials in case $n=m$, see [10] for a proof and historic overview. In (1.1) we require $n, m \in \mathbb{N}, \Re \nu>-1, y>0$, $\sigma \in \mathbb{C}$, and we use the standard notation for Laguerre polynomials and Bessel functions as in e.g. [5]. There are many relations between Laguerre polynomials and the Hankel transform, the most well-known being that the Laguerre polynomials arise as the eigenfunctions of the Hankel transform. For some of these identities there is a group theoretic interpretation, but as far as we know this is not the case for Erdélyi's identity (1.1).

We present a $q$-analogue of Erdélyi's identity, which surprisingly does have a quantum group theoretic derivation. The essential ingredient is the use of the linking quantum groupoid between the quantum group analogues of $S U(2)$ and the double cover of $E(2)$, the group of plane motions. This linking quantum groupoid is studied extensively in [1], 2], and we recall the necessary results in Section 4.

In some sense the result is reminiscent of the quantum group theoretic derivation of the addition formula for the little $q$-Legendre polynomials by Koornwinder [1] and Graf's addition formula for the ${ }_{1} \varphi_{1} q$-Bessel functions [7, §6], which in turn is motivated by [11. The linking quantum groupoid allows us to connect these two developments, and we obtain the $q$-analogue of (1.1) from this connection.

Received by the editors August 13, 2013 and, in revised form, January 14, 2014.

2010 Mathematics Subject Classification. Primary 33D80, 33D45, 46L65, 81R50. 
The contents of the paper are as follows. In Sections 2 and 3 we recall the necessary preliminaries on special functions and quantum groups, and we recall as well the definition of the standard Podleś sphere. In Section 4 we present the above mentioned quantum linking groupoid and its comultiplication, which is employed in Section 5 to derive a $q$-analogue of (1.1). Having the result at hand we can then extend various of the parameters to a more general domain. The $q$-Hankel transform is as in 13, and taking the inverse gives the same formula. In Remark 5.2 (ii) we sketch an analytic, though verificational, proof.

Koornwinder's addition formula [1] has been generalized to Askey-Wilson polynomials in 8] using Koornwinder's [12 twisted primitive elements in quantum groups, and we expect that Theorem 5.1 can be extended in a similar way to the level of the Askey-Wilson Hankel transform, see [9, Fig. 2.1]. From [1] we know that there is also a quantum linking groupoid between the quantum group analogues of $S U(2)$ and the normalizer of $S U(1,1)$ in $S L(2, \mathbb{C})$, the construction of which involves non-standard Podleś spheres. It might be possible to obtain a similar result in this case even though the unitary operator implementing the comultiplication becomes more involved.

\section{Preliminaries: $q$-SPECial FunCtions}

In this section we recall the $q$-special functions needed, and we fix the notation.

2.1. Basic hypergeometric series. We follow the notation for basic hypergeometric series in Gasper and Rahman [4, see also e.g. [5], 6]. Explicitly, we assume $0<q<1$ and define the $q$-shifted factorials for $a \in \mathbb{C}, k \in \mathbb{N}$ by

$$
(a ; q)_{k}=\prod_{i=0}^{k-1}\left(1-a q^{i}\right), \quad(a ; q)_{\infty}=\lim _{k \rightarrow \infty}(a ; q)_{k}, \quad\left(a_{1}, \cdots, a_{r} ; q\right)_{k}=\prod_{i=1}^{r}\left(a_{i} ; q\right)_{k}
$$

also allowing $k=\infty$ in the last definition. Then the basic (or $q$-)hypergeometric series is

$$
{ }_{r} \varphi_{s}\left(\begin{array}{c}
a_{1}, \cdots, a_{r} \\
b_{1}, \cdots b_{s}
\end{array} ; q, z\right)=\sum_{k=0}^{\infty} \frac{\left(a_{1}, \cdots, a_{r} ; q\right)_{k}}{\left(b_{1}, \cdots, b_{s} ; q\right)_{k}} \frac{z^{k}}{(q ; q)_{k}}\left((-1)^{k} q^{\frac{1}{2} k(k-1)}\right)^{1+s-r} .
$$

We assume that $b_{i} \notin q^{-\mathbb{N}}$ for all $i$. The series terminates if $a_{i} \in q^{-\mathbb{N}}$ for some $i$. In general, the radius of convergence is

- 1 in case $r=s+1$,

- $\infty$ if $1+s>r$, and

- 0 if $1+s<r$.

Note however that

$$
\left(b_{1} ; q\right)_{\infty r} \varphi_{s}\left(\begin{array}{c}
a_{1}, \cdots, a_{r} \\
b_{1}, \cdots b_{s}
\end{array} ; q, z\right)
$$

is analytic in $b_{1}$, and there is no need to exclude $q^{-\mathbb{N}}$ for $b_{1}$. In case $b_{1}=q^{1-n}$, $n \in \mathbb{N}$, the summation starts at $k=n$. 
2.2. Wall polynomials. The Wall polynomials are defined as a subclass of the little $q$-Jacobi polynomials, see [4], 6]. Explicitly, we define the Wall polynomials for $0<a<q^{-1}$ as

$$
\begin{aligned}
p_{n}(x ; a ; q) & ={ }_{2} \varphi_{1}\left(\begin{array}{c}
q^{-n}, 0 \\
a q
\end{array} ; q, q x\right) \\
& =\frac{(-1)^{n} q^{\frac{1}{2} n(n+1)}(a x)^{n}}{(a q ; q)_{n}}{ }_{3} \varphi_{2}\left(\begin{array}{c}
q^{-n}, q^{-n} / a, 1 / x \\
0,0
\end{array} ; q, q\right)
\end{aligned}
$$

see [11, (2.8)]. The Wall polynomials are $q$-analogues of the Laguerre polynomials in view of

$$
\lim _{q \uparrow 1} p_{n}\left((1-q) x ; q^{\alpha} ; q\right)=\frac{L_{n}^{(\alpha)}(x)}{L_{n}^{(\alpha)}(0)},
$$

where the notation for Laguerre polynomials $L_{n}^{(\alpha)}(x)$ is the standard notation as in e.g. [5, §4.6], [6, §1.11]. The orthogonality relations and the dual orthogonality relations for the Wall polynomials are

$$
\begin{aligned}
& \sum_{k=0}^{\infty} \frac{(a q)^{k}}{(q ; q)_{k}} p_{n}\left(q^{k} ; a ; q\right) p_{m}\left(q^{k} ; a ; q\right)=\delta_{n m} \frac{(a q)^{n}(q ; q)_{n}}{(a q ; q)_{n}(a q ; q)_{\infty}}, \\
& \sum_{n=0}^{\infty} \frac{(a q ; q)_{n}}{(a q)^{n}(q ; q)_{n}} p_{n}\left(q^{k} ; a ; q\right) p_{n}\left(q^{l} ; a ; q\right)=\delta_{k l} \frac{(a q)^{-k}(q ; q)_{k}}{(a q ; q)_{\infty}}
\end{aligned}
$$

where the dual orthogonality relations correspond to the orthogonality relations for the Al-Salam-Carlitz II polynomials, see [6], [11, §2], [14, Prop. 3.3].

2.3. ${ }_{1} \varphi_{1} q$-Bessel functions. For the ${ }_{1} \varphi_{1} q$-Bessel functions (also known as Jackson's third $q$-Bessel function or as the Hahn-Exton $q$-Bessel function) we follow Koornwinder and Swarttouw [13. Define the ${ }_{1} \varphi_{1} q$-Bessel function of order $\nu$

$$
J_{\nu}(x ; q)=x^{\nu} \frac{\left(q^{\nu+1} ; q\right)_{\infty}}{(q ; q)_{\infty}}{ }_{1} \varphi_{1}\left(\begin{array}{c}
0 \\
q^{\nu+1}
\end{array} ; q, q x^{2}\right) .
$$

Note that this is well-defined for $\nu=n \in \mathbb{Z}$, see 2.1 and in this case we have $J_{-n}\left(w ; q^{2}\right)=(-q)^{n} J_{n}\left(w q^{n} ; q^{2}\right)$. We also have the symmetry $J_{\nu}\left(q^{\alpha} ; q^{2}\right)=$ $J_{\alpha}\left(q^{\nu} ; q^{2}\right)$, see [13, Prop. 2.1]. Moreover, we have $\lim _{q \uparrow 1} J_{\nu}\left((1-q) x ; q^{2}\right)=J_{\nu}(x)$, where $J_{\nu}(\cdot)$ is the Bessel function, see [13, §3]. The ${ }_{1} \varphi_{1} q$-Bessel functions can be obtained from the little $q$-Jacobi polynomials, see [13, §3], and the following orthogonality relations hold:

$$
\sum_{k \in \mathbb{Z}} q^{k+n} J_{k+n}\left(q^{l} ; q^{2}\right) q^{k+m} J_{k+m}\left(q^{l} ; q^{2}\right)=\delta_{n m}, \quad n, m, l \in \mathbb{Z} .
$$

The orthogonality relations can also be rewritten as a $q$-Hankel transform pair:

$$
g\left(q^{n}\right)=\sum_{k=-\infty}^{\infty} q^{2 k} J_{\nu}\left(q^{k+n} ; q^{2}\right) f\left(q^{k}\right) \Leftrightarrow f\left(q^{k}\right)=\sum_{n=-\infty}^{\infty} q^{2 n} J_{\nu}\left(q^{k+n} ; q^{2}\right) g\left(q^{n}\right)
$$

for $f$ and $g$ square integrable on $q^{\mathbb{Z}}$ with respect to the counting measure, $n, k \in \mathbb{Z}$, see [13, §3]. Note that (2.6) can also be written using Jackson's $q$-integral,

$$
\int_{0}^{\infty} f(x) d_{q} x=(1-q) \sum_{k=-\infty}^{\infty} f\left(q^{k}\right) q^{k}
$$

for any function so that the sum converges. 


\section{Preliminaries: quantum groups and Podleś sphere}

In this section we introduce the quantum group analogues of $S U(2)$ and the double cover $\widetilde{E}(2)$ of the group of plane motions $E(2)$, as well as the standard Podleś sphere, on the level of von Neumann algebras. The main tools are the comultiplication and the corresponding action on the Podleś sphere. See [1], 2] for precise information and references.

3.1. The quantum $S U(2)$ group. The quantum $S U(2)$ group is one of the earliest and most basic examples of a quantum group. It has been introduced by Woronowicz [18, see also [17, §6.2] and references. The harmonic analysis and the relation with $q$-special functions on the quantum $S U(2)$ group is of great interest.

Definition 3.1. Let $0<q<1$. The Hopf $*$-algebra $\left(\operatorname{Pol}\left(S U_{q}(2)\right), \Delta_{+}\right)$is the universal unital $*$-algebra generated by elements $\alpha, \gamma$ which make the matrix $u=$ $\left(\begin{array}{cc}\alpha & -q \gamma^{*} \\ \gamma & \alpha^{*}\end{array}\right)$ a unitary corepresentation.

Definition 3.1 is a compact formulation due to Woronowicz's result [18, Thm. 1.4]. In particular, the Hopf $*$-algebra $\operatorname{Pol}\left(S U_{q}(2)\right)$ is generated by $\alpha$ and $\gamma$ subject to the relations

$$
\alpha \gamma=q \gamma \alpha, \quad \alpha \gamma^{*}=q \gamma^{*} \alpha, \quad \gamma^{*} \gamma=\gamma \gamma^{*}, \quad \alpha^{*} \alpha+\gamma^{*} \gamma=1=\alpha \alpha^{*}+q^{2} \gamma^{*} \gamma
$$

and with comultiplication $\Delta_{+}: \operatorname{Pol}\left(S U_{q}(2)\right) \rightarrow \operatorname{Pol}\left(S U_{q}(2)\right) \otimes \operatorname{Pol}\left(S U_{q}(2)\right)$ given by

$$
\Delta_{+}(\alpha)=\alpha \otimes \alpha-q \gamma^{*} \otimes \gamma, \quad \Delta_{+}(\gamma)=\gamma \otimes \alpha+\alpha^{*} \otimes \gamma
$$

Associated to the Hopf $*$-algebra $\left(\operatorname{Pol}\left(S U_{q}(2)\right), \Delta_{+}\right)$there is a von Neumann bialgebra $\left(\mathscr{L}^{\infty}\left(S U_{q}(2)\right), \Delta_{+}\right)$, which we now describe. We use the notation $\mathscr{L}(\mathbb{Z})$ to denote the group von Neumann algebra of $\mathbb{Z}$, i.e. the von Neumann algebra generated by the bilateral unitary shift operator $S: e_{n} \mapsto e_{n+1}$ acting on $\ell^{2}(\mathbb{Z})$, where the $e_{n}$ denote the standard orthonormal basis. In general, we will denote $e_{m, n, \ldots}$ for $e_{m} \otimes e_{n} \otimes \ldots$ as an element in a tensor product of $\ell^{2}$-spaces.

The explicit implementation of the comultiplication $\Delta_{+}$in the following Definition-Proposition 3.2 is due to Koornwinder [11, Lance [14]. The formulation is taken from [1, App. A].

Definition-Proposition 3.2. The von Neumann bialgebra $\left(\mathscr{L}^{\infty}\left(S U_{q}(2)\right), \Delta_{+}\right)$ has as its underlying von Neumann algebra $\mathscr{L}^{\infty}\left(S U_{q}(2)\right)=B\left(\ell^{2}(\mathbb{N})\right) \bar{\otimes} \mathscr{L}(\mathbb{Z})$, where $\bar{\otimes}$ denotes the spatial tensor product of von Neumann algebras. The comultiplication is given by $\Delta_{+}(x)=W_{+}^{*}(1 \otimes x) W_{+}$, where $W_{+}$is a unitary map defined by

$$
\begin{gathered}
W_{+}:\left(\ell^{2}(\mathbb{N}) \otimes \ell^{2}(\mathbb{Z})\right) \otimes\left(\ell^{2}(\mathbb{N}) \otimes \ell^{2}(\mathbb{Z})\right) \rightarrow\left(\ell^{2}(\mathbb{Z}) \otimes \ell^{2}(\mathbb{Z})\right) \otimes\left(\ell^{2}(\mathbb{N}) \otimes \ell^{2}(\mathbb{Z})\right) \\
\xi_{r, s, p, t}^{+} \mapsto e_{r, s, p, t}, \quad p \in \mathbb{N}, r, s, t \in \mathbb{Z},
\end{gathered}
$$


where $\left\{\xi_{r, s, p, t}^{+} \mid p \in \mathbb{N}, r, s, t \in \mathbb{Z}\right\}$ is an orthonormal basis of $\left(\ell^{2}(\mathbb{N}) \otimes \ell^{2}(\mathbb{Z})\right) \otimes$ $\left(\ell^{2}(\mathbb{N}) \otimes \ell^{2}(\mathbb{Z})\right)$ defined by

$$
\begin{array}{r}
\xi_{r, s, p, t}^{+}=\sum_{\substack{v, w \in \mathbb{N} \\
v-w=t}} P^{+}(p, v, w) e_{v, r+p-w, w, s-p+v} \\
P^{+}(p, v, w)=(-q)^{p-w} q^{(p-w)(v-w)} \sqrt{\frac{\left(q^{2 p+2}, q^{2 w+2} ; q^{2}\right)_{\infty}}{\left(q^{2 v+2} ; q^{2}\right)_{\infty}}} \\
\quad \times \frac{\left(q^{2 v-2 w+2} ; q^{2}\right)_{\infty}}{\left(q^{2} ; q^{2}\right)_{\infty}} p_{w}\left(q^{2 p} ; q^{2 v-2 w} ; q^{2}\right)
\end{array}
$$

for $p, v, w \in \mathbb{N}$.

Remark 3.3. (i) The fact that $\left\{\xi_{r, s, p, t}^{+}\right\}_{p \in \mathbb{N}, r, s, t \in \mathbb{Z}}$ is an orthonormal basis for the space $\left(\ell^{2}(\mathbb{N}) \otimes \ell^{2}(\mathbb{Z})\right) \otimes\left(\ell^{2}(\mathbb{N}) \otimes \ell^{2}(\mathbb{Z})\right)$, i.e. that $W_{+}$is a unitary operator, is equivalent to the orthogonality and dual orthogonality relations (2.3) for the Wall polynomials, which can be stated as

$$
\begin{aligned}
& \sum_{p=0}^{\infty} P^{+}(p, v, w) P^{+}\left(p, v^{\prime}, w^{\prime}\right)=\delta_{v, v^{\prime}} \quad \text { if } v-w=v^{\prime}-w^{\prime}, \\
& \sum_{\substack{v, w \in \mathbb{N} \\
v-w=t}} P^{+}(p, v, w) P^{+}\left(p^{\prime}, v, w\right)=\delta_{p, p^{\prime}} .
\end{aligned}
$$

(ii) Using the explicit expression (2.2) for the Wall polynomial as a ${ }_{3} \varphi_{2}$-function we find that $P^{+}(p, v, w)(-q)^{-p}$ is symmetric in $p, v$ and $w$. The expression for $P^{+}(p, v, w)$ coincides with the notation as in [1, Def. 0.4, App. A]. The identification with [11, (2.5)] is $P^{+}(p, v, w)=(-1)^{p} P_{v}\left(q^{2 p} ; q^{2(w-v)} \mid q^{2}\right)$.

(iii) The embedding of $\operatorname{Pol}\left(S U_{q}(2)\right)$ into $\mathscr{L}^{\infty}\left(S U_{q}(2)\right)$ is given by

$$
\alpha e_{n, k}=\sqrt{1-q^{2 n}} e_{n-1, k}, \quad \gamma e_{n, k}=q^{n} e_{n, k+1},
$$

with the convention $e_{-1}=0$ in $\ell^{2}(\mathbb{N})$, so that $e_{-1, k}=0$. The fact that the comultiplication (3.2) agrees with Definition-Proposition 3.2 follows from suitable contiguous relations for the Wall polynomials, hence the $P^{+}$-functions, see [1, App. A]. (In 2004 Groenevelt (unpublished notes) obtained the same result.) For this the sign-difference with the notation of Koornwinder [11] is essential.

(iv) A straightforward calculation shows that with respect to the standard basis we have

$$
W_{+} e_{m, k, n, l}=\sum_{p \in \mathbb{N}} P^{+}(p, m, n) e_{k+n-p, l-m+p, p, m-n} .
$$

\subsection{The quantum $\widetilde{E}(2)$ group.}

Definition-Proposition 3.4. The von Neumann bialgebra $\left(\mathscr{L}^{\infty}\left(\widetilde{E}_{q}(2)\right), \Delta_{0}\right)$ has as its associated von Neumann algebra $\mathscr{L}^{\infty}\left(\widetilde{E}_{q}(2)\right)=B\left(\ell^{2}(\mathbb{Z})\right) \bar{\otimes} \mathscr{L}(\mathbb{Z})$. The comultiplication $\Delta_{0}(x)=W_{0}^{*}(1 \otimes x) W_{0}$ for all $x \in \mathscr{L}^{\infty}\left(\widetilde{E}_{q}(2)\right)$ is defined by the unitary map

$$
\begin{gathered}
W_{0}:\left(\ell^{2}(\mathbb{Z}) \otimes \ell^{2}(\mathbb{Z})\right) \otimes\left(\ell^{2}(\mathbb{Z}) \otimes \ell^{2}(\mathbb{Z})\right) \rightarrow\left(\ell^{2}(\mathbb{Z}) \otimes \ell^{2}(\mathbb{Z})\right) \otimes\left(\ell^{2}(\mathbb{Z}) \otimes \ell^{2}(\mathbb{Z})\right) \\
\xi_{r, s, p, t}^{0} \mapsto e_{r, s, p, t},
\end{gathered}
$$


where $\left\{\xi_{r, s, p, t}^{0} \mid r, s, p, t \in \mathbb{Z}\right\}$ is the orthonormal basis of $\left(\ell^{2}(\mathbb{Z}) \otimes \ell^{2}(\mathbb{Z})\right) \otimes\left(\ell^{2}(\mathbb{Z}) \otimes\right.$ $\left.\ell^{2}(\mathbb{Z})\right)$ defined by

$$
\xi_{r, s, p, t}^{0}=\sum_{\substack{v, w \in \mathbb{Z} \\ v-w=t}} P^{0}(p, v, w) e_{v, r+p-w, w, s-p+v}
$$

for $p, r, s, t \in \mathbb{Z}$, where $P^{0}(p, v, w)=(-q)^{p-w} J_{v-w}\left(q^{p-w} ; q^{2}\right)$ for $p, v, w \in \mathbb{Z}$.

Remark 3.5. (i) Using the explicit expression (2.4) and the symmetries for the ${ }_{1} \varphi_{1} q$-Bessel functions as in [13, Prop. 2.1, (2.6)] we find that $P^{0}(p, v, w)(-q)^{-p}$ is symmetric in $p, v$ and $w$. The expression for $P^{0}(p, v, w)$ coincides with the notation as in [1, Prop. 0.6, App. A]. From (2.5) and the symmetries for the ${ }_{1} \varphi_{1} q$-Bessel functions we find that (2.5) gives

$$
\begin{aligned}
& \sum_{p=-\infty}^{\infty} P^{0}(p, v, w) P^{0}\left(p, v^{\prime}, w^{\prime}\right)=\delta_{v, v^{\prime}} \quad \text { if } v-w=v^{\prime}-w^{\prime}, \\
& \sum_{\substack{v, w \in \mathbb{Z} \\
v-w=t}} P^{0}(p, v, w) P^{0}\left(p^{\prime}, v, w\right)=\delta_{p, p^{\prime}} .
\end{aligned}
$$

Note that (3.5) are equivalent to $\left\{\xi_{r, s, p, t}^{0}\right\}_{r, p, s, t \in \mathbb{Z}}$ being an orthonormal basis, hence $W_{0}$ is a unitary operator. See [1, Prop. 4.2, App. A] for the implementation of the comultiplication, and also 7 where the implementation is implicit.

(ii) Define

$$
v e_{m, k}=e_{m-1, k}, \quad v \in \mathscr{L}^{\infty}\left(\widetilde{E}_{q}(2)\right),
$$

and let $n$ denote the unique unbounded normal operator affiliated with $\mathscr{L}^{\infty}\left(\widetilde{E}_{q}(2)\right)$ satisfying

$$
n e_{m, k}=q^{m} e_{m, k+1} .
$$

Then $v$ and $n$ are the generators of the quantum group $\widetilde{E}_{q}(2)$ as introduced by Woronowicz [19]. In particular,

$$
\Delta_{0}(v)=W_{0}^{*}(1 \otimes v) W_{0}=v \otimes v .
$$

as follows from $P^{0}(p, v, w)=P^{0}(p-1, v-1, w-1)$, see [1, App. A] for more information.

(iii) Note that $\lim _{N \rightarrow \infty} P^{+}(p+N, v+N, w+N)=P^{0}(p, v, w)$, and then (3.3) go over into (3.5), cf. [13, $\S 3$, App. A]. One may consider this limit as a reflection of the contraction procedure of the quantum $S U(2)$ group to the quantum $\tilde{E}(2)$ group, see [20, §2].

3.3. The standard Podleś sphere. The Podleś spheres are a 1-parameter family of quantum analogues of the 2-sphere originally introduced in [15]. We need one particular case, see [1] for more details.

Definition 3.6. Let $0<q<1$. The ${ }^{*}$-algebra $\operatorname{Pol}\left(S_{q}^{2}\right)$ is generated by elements $X, Y$ and $Z$ satisfying $X^{*}=Y, Z^{*}=Z$ and

$$
X Z=q^{2} Z X, \quad Y Z=q^{-2} Z Y, \quad X Y=Z-q^{2} Z^{2}, \quad Y X=Z-q^{-2} Z^{2} .
$$

Then $\operatorname{Pol}\left(S_{q}^{2}\right)$ is the polynomial $*$-algebra associated to the standard Podles sphere $S_{q}^{2}$. The algebra $\operatorname{Pol}\left(S_{q}^{2}\right)$ carries a coaction $\Upsilon: \operatorname{Pol}\left(S_{q}^{2}\right) \rightarrow \operatorname{Pol}\left(S U_{q}(2)\right) \otimes \operatorname{Pol}\left(S_{q}^{2}\right)$, 
determined by

$$
\begin{gathered}
\Upsilon\left(\begin{array}{c}
X \\
1-\left(1+q^{2}\right) Z \\
Y
\end{array}\right)= \\
\left(\begin{array}{ccc}
\alpha^{2} & -\gamma^{*} \alpha & -q\left(\gamma^{*}\right)^{2} \\
\left(1+q^{2}\right) \alpha \gamma & 1-\left(1+q^{2}\right) \gamma^{*} \gamma & \left(1+q^{2}\right) \gamma^{*} \alpha^{*} \\
-q \gamma^{2} & -\alpha^{*} \gamma & \left(\alpha^{*}\right)^{2}
\end{array}\right) \otimes\left(\begin{array}{c}
X \\
1-\left(1+q^{2}\right) Z \\
Y
\end{array}\right) .
\end{gathered}
$$

Remark 3.7. (i) The map $\phi: \operatorname{Pol}\left(S_{q}^{2}\right) \rightarrow \operatorname{Pol}\left(S U_{q}(2)\right)$ defined by

$$
X \mapsto-\gamma^{*} \alpha \quad Z \mapsto \gamma^{*} \gamma \quad Y \mapsto-\alpha^{*} \gamma
$$

embeds $\operatorname{Pol}\left(S_{q}^{2}\right)$ as a left coideal in $\operatorname{Pol}\left(S U_{q}(2)\right)$. The embedding is equivariant: $($ id $\otimes \phi) \Upsilon=\Delta_{+} \phi$.

(ii) The operators on $\ell^{2}(\mathbb{N})$ defined by

$$
X e_{n}=-q^{n-1} \sqrt{1-q^{2 n}} e_{n-1}, \quad Z e_{n}=q^{2 n} e_{n}, \quad Y e_{n}=-q^{n} \sqrt{1-q^{2 n+2}} e_{n+1}
$$

represent $\operatorname{Pol}\left(S_{q}^{2}\right)$ faithfully. Set $U: e_{n, k} \mapsto e_{n, n+k}$, so that $U \in B\left(\ell^{2}(\mathbb{N}) \otimes \ell^{2}(\mathbb{Z})\right)$ is unitary. Then (3.8) and (3.4) are related by $U$, so $U(x \otimes 1) U^{*}=\phi(x)$ for all $x \in \operatorname{Pol}\left(S_{q}^{2}\right)$.

\section{Quantum Linking GRoupoid}

The quantum linking groupoid relates the quantum group analogues of $S U(2)$ and the double cover of $E(2)$ as given in Definition-Propositions 3.2 and 3.4 . In order to describe the construction briefly we start with an explicit realisation of the Podleś sphere, see [1, 2] for more information.

\subsection{Implementation of the Podleś sphere and quantum linking groupoid.} We define

$$
N=B\left(\ell^{2}(\mathbb{N}), \ell^{2}(\mathbb{Z})\right) \bar{\otimes} \mathscr{L}(\mathbb{Z}) \subset B\left(\ell^{2}(\mathbb{N}) \otimes \ell^{2}(\mathbb{Z}), \ell^{2}(\mathbb{Z}) \otimes \ell^{2}(\mathbb{Z})\right)
$$

and we equip it with the normal linear map

$$
\Delta_{0+}: N \rightarrow N \bar{\otimes} N, \quad \Delta_{0+}(x)=W_{0}^{*}(1 \otimes x) W_{+} \quad x \in N,
$$

which is easily seen to be well-defined using the bicommutant theorem.

Then $\Delta_{0+}$ can be shown to be coassociative, and to turn $N$ into a "linking bimodule coalgebra" between $\mathscr{L}^{\infty}\left(\tilde{E}_{q}(2)\right)$ and $\mathscr{L}^{\infty}\left(S U_{q}(2)\right)$. Together with the space of adjoint operators and the function algebras on the two quantum groups, it can be considered as a quantum groupoid, called "linking quantum groupoid", see [1, 2].

Definition-Proposition 4.1. [2, §3] Define the unitary operator

$$
\begin{gathered}
\mathcal{G}: \ell^{2}(\mathbb{N}) \otimes \ell^{2}(\mathbb{Z}) \otimes \ell^{2}(\mathbb{N}) \rightarrow \ell^{2}(\mathbb{Z}) \otimes \ell^{2}(\mathbb{Z}) \otimes \ell^{2}(\mathbb{N}) \\
\mathcal{G}: \eta_{r, p, t} \mapsto e_{t-p, r, p}, \quad \eta_{r, p, t}=\sum_{\substack{v, w \in \mathbb{N} \\
v-w=t}} P^{+}(p, v, w) e_{v, r+p-w, w},
\end{gathered}
$$


where $p \in \mathbb{N}, r, t \in \mathbb{Z}$. Then $\mathcal{G} \in N \bar{\otimes} B\left(\ell^{2}(\mathbb{N})\right)$ and $\mathcal{G}$ implements $\Upsilon: \operatorname{Pol}\left(S_{q}^{2}\right) \rightarrow$ $\operatorname{Pol}\left(S U_{q}(2)\right) \otimes \operatorname{Pol}\left(S_{q}^{2}\right)$, i.e.

$$
\Upsilon(x)=\mathcal{G}^{*}(1 \otimes x) \mathcal{G}, \quad \forall x \in \operatorname{Pol}\left(S_{q}^{2}\right) .
$$

Moreover, $\left(\Delta_{0+} \otimes \mathrm{id}\right)(\mathcal{G})=\mathcal{G}_{13} \mathcal{G}_{23}$.

The last formula of Proposition 4.1 means that $\mathcal{G}$ is to be seen as a unitary projective corepresentation of $\mathscr{L}^{\infty}\left(S U_{q}(2)\right)$. Together with the explicit implementation of the comultiplication $\Delta_{0+}$ it is the crucial formula for this paper. In Section 5 this identity is converted into a $q$-analogue of Erdélyi's formula. This formula follows from the general construction as described in [2, §2] introduced to study Morita equivalence of quantum groups.

We can expand $\mathcal{G}$ by

$$
\mathcal{G}=\sum_{n, m \in \mathbb{N}} \mathcal{G}_{m n} \otimes e_{m n}, \quad \mathcal{G}_{m n}: e_{a, b} \mapsto P^{+}(m, a, n) e_{a-n-m, b+n-m},
$$

where $e_{m n} \in B\left(\ell^{2}(\mathbb{N})\right)$ is the matrix-unit defined by $e_{m n} e_{k}=\delta_{n k} e_{m}$.

Finally, we define

$$
\mathcal{G}_{m n}^{(l)}=v^{l} \mathcal{G}_{m n} \in B\left(\ell^{2}(\mathbb{N}) \otimes \ell^{2}(\mathbb{Z}), \ell^{2}(\mathbb{Z}) \otimes \ell^{2}(\mathbb{Z})\right), \quad \mathcal{G}^{(l)}=\sum_{m, n \in \mathbb{N}} \mathcal{G}_{m n}^{(l)} \otimes e_{m n}
$$

where $v$ is the shift operator (3.6). Then Proposition 4.1 and (3.7) imply

$$
\left(\Delta_{0+} \otimes \mathrm{id}\right)\left(\mathcal{G}^{(l)}\right)=\mathcal{G}_{13}^{(l)} \mathcal{G}_{23}^{(l)} .
$$

Moreover, from (4.2) and (3.6) we find

$$
\mathcal{G}_{m n}^{(l)}: \ell^{2}(\mathbb{N}) \otimes \ell^{2}(\mathbb{Z}) \rightarrow \ell^{2}(\mathbb{Z}) \otimes \ell^{2}(\mathbb{Z}), \quad \mathcal{G}_{m n}^{(l)}: e_{a, b} \mapsto P^{+}(m, a, n) e_{a-l-n-m, b+n-m} .
$$

\section{A q-ANAlogue of ERdélyi's formula}

The goal of this section is to prove a first $q$-analogue of Erdélyi's formula (1.1) on the lowest level of the $q$-scheme as discussed in [9, Fig. 1.2, §4].

Theorem 5.1. Recall the notation of (2.2), (2.4) for Wall polynomials and little $q$-Bessel functions. Write $\tilde{p}_{n}(x ; a ; q)=(q a ; q)_{n} p_{n}(x ; a ; q)$. Then we have

$$
\begin{aligned}
& \sum_{p=0}^{\infty} q^{2 p} q^{p \nu}\left(q^{2+2 p} ; q^{2}\right)_{\infty} \tilde{p}_{n}\left(q^{2 p} ; q^{2 \sigma} ; q^{2}\right) \tilde{p}_{m}\left(q^{2 p} ; q^{2(\nu-\sigma)} ; q^{2}\right) J_{\nu}\left(z q^{p} ; q^{2}\right) \\
& =(-q)^{n+m} z^{\nu} q^{(m-n)^{2}} q^{2 n \sigma+2 m(\nu-\sigma)}\left(z^{2} q^{2(1+n+m)} ; q^{2}\right)_{\infty} \\
& \quad \times \tilde{p}_{n}\left(z^{2} q^{2(n+m)} ; q^{2(\nu-\sigma+m-n)} ; q^{2}\right) \tilde{p}_{m}\left(z^{2} q^{2(n+m)} ; q^{2(\sigma+n-m)} ; q^{2}\right),
\end{aligned}
$$

where $\Re \nu>-1, z \in \mathbb{C}$ with $|\arg z|<\pi, n, m \in \mathbb{N}, \sigma \in \mathbb{C}$.

Remark 5.2. (i) Write $E_{q^{2}}(z)=\left(-z ; q^{2}\right)_{\infty}$ for the big $q$-exponential function 4, $\S 1.3]$, and write $\check{p}_{n}(x ; a ; q)=(q a ; q)_{\infty} p_{n}(x ; a ; q)$. Then using the notation (2.7), we can rewrite the result of Theorem 5.1 as

$$
\begin{aligned}
& \frac{1}{1-q} \int_{0}^{\infty} x^{\nu} E_{q^{2}}\left(-q^{2} x^{2}\right) \check{p}_{n}\left(x^{2} ; q^{2 \sigma} ; q^{2}\right) \check{p}_{m}\left(x^{2} ; q^{2(\nu-\sigma)} ; q^{2}\right) J_{\nu}\left(z x ; q^{2}\right) x d_{q} x \\
& =(-q)^{n+m} z^{\nu} q^{(m-n)^{2}} q^{2 n \sigma+2 m(\nu-\sigma)} E_{q^{2}}\left(-z^{2} q^{2(1+n+m)}\right) \\
& \quad \times \check{p}_{n}\left(z^{2} q^{2(n+m)} ; q^{2(\nu-\sigma+m-n)} ; q^{2}\right) \check{p}_{m}\left(z^{2} q^{2(n+m)} ; q^{2(\sigma+n-m)} ; q^{2}\right) .
\end{aligned}
$$


Replacing $x, z$ by $x \sqrt{1-q}, z \sqrt{1-q}$ and using the $q$-Gamma function, we see that we can take the formal limit $q \uparrow 1$ of (5.1) to (1.1).

(ii) The special case $n=m=0$ is

$$
\sum_{p=0}^{\infty} q^{2 p} q^{p \nu}\left(q^{2+2 p} ; q^{2}\right)_{\infty} J_{\nu}\left(z q^{p} ; q^{2}\right)=z^{\nu}\left(z^{2} q^{2} ; q^{2}\right)_{\infty},
$$

which is directly proved using the $q$-binomial formula and the big $q$-exponential function, see [4, §1.3], after using (2.4) and interchanging summations. In the same spirit we can evaluate the left hand side of Theorem 5.1 in the case $m=0$, which gives a series expansion in $z$ (up to $z^{\nu}$ ). Expanding the right hand side of Theorem 5.1 in the same way, we see that we need the $q$-Chu-Vandermonde summation [4. (1.5.3)] to establish equality. The case for arbitrary degrees $m$ can be obtained by induction with respect to $m$, and using the three-term recurrence relation for the Wall polynomials both in the degree $n$ as in $m$. Note that this is a verificational proof, and completely different in spirit from e.g. Rahman's [16 analytic proof of Koornwinder's [11] addition formula for the little $q$-Legendre polynomials. It would be desirable to have an analytic proof of Theorem 5.1 in the style of Rahman [16. (iii) We need the additional freedom of $l$ introduced in (4.3) in order to get the result of Theorem 5.1 in full generality.

(iv) Theorem 5.1 can be viewed as a $q$-Hankel transform. The inverse, see [13, (3.4)], can be written down, and the resulting inverse identity is equivalent to Theorem 5.1 for $z=q^{r}, r \in \mathbb{Z}$.

Proof of Theorem 5.1. We start with the corepresentation property of $\mathcal{G}^{(l)}$, see (4.4), in combination with the implementation of the comultiplication as in Proposition 4.1 to find the operator identity

$$
W_{0,12}^{*} \mathcal{G}_{23}^{(l)} W_{+, 12}=\mathcal{G}_{13}^{(l)} \mathcal{G}_{23}^{(l)}:\left(\ell^{2}(\mathbb{N}) \otimes \ell^{2}(\mathbb{Z})\right)^{\otimes 2} \otimes \ell^{2}(\mathbb{N}) \rightarrow\left(\ell^{2}(\mathbb{Z})\right)^{\otimes 4} \otimes \ell^{2}(\mathbb{N})
$$

Since (5.3) is an identity for operators, we let (5.3) act on the basis vector $e_{a, b, c, d, e}$, $a, c, e \in \mathbb{N}, b, d \in \mathbb{Z}$. The action of the left hand side of (5.3) on this basis vector can be calculated using Remark 3.3(iv), (4.5) and Definition-Proposition 3.4, and we find

$$
\sum_{p, m \in \mathbb{N}} P^{+}(p, a, c) P^{+}(m, p, e) \xi_{b+c-p, d-a+p, p-l-m-e, a-c+e-m}^{0} \otimes e_{m},
$$

with $\xi_{\text {rspt }}^{0}$ as in Definition-Propostion 3.4. The action of the right hand side of (5.3) on this basis vector can be calculated using (4.5) and we find

$$
\sum_{m, r \in \mathbb{N}} P^{+}(m, c, e) P^{+}(r, a, m) e_{a-l-r-m, b-r+m, c-l-e-m, d+e-m, r} .
$$

Since (5.3) leads to the equality of (5.4) and (5.5) as identity in $\ell^{2}(\mathbb{Z})^{\otimes 4} \otimes \ell^{2}(\mathbb{N})$, we can take inner products with an arbitrary basis vector $e_{u, v, w, x, y} \in \ell^{2}(\mathbb{Z})^{\otimes 4} \otimes$ $\ell^{2}(\mathbb{N})$ to obtain a scalar identity. A calculation shows that the inner product with (5.5) gives

$$
P^{+}(d+e-x, c, e) P^{+}(y, a, d+e-x)
$$

in case

$$
d+e-x=c-l-e-w=-b+y+v=a-l-y-u \in \mathbb{N},
$$


and 0 otherwise. A calculation shows that the inner product with (5.4) gives

$$
\sum_{p=0}^{\infty} P^{+}(p, a, c) P^{+}(y, p, e) P^{0}(p-l-y-e, a-c+e-y+w, w) .
$$

in case $u-w=a-c+e-y, v+w=b+c-l-y-e$, and $x-u=d+y+e-a+l$, and 0 otherwise. Assuming these conditions, which match the equalities in (5.6), and eliminating superfluous parameters we obtain

$$
\begin{aligned}
& \sum_{p=0}^{\infty} P^{+}(p, a, c) P^{+}(y, p, e) P^{0}(p-l-y-e, a-c+e-y+w, w) \\
& = \begin{cases}P^{+}(c-l-e-w, c, e) P^{+}(y, a, c-l-e-w), & c-l-e-w \in \mathbb{N} \\
0, & c-l-e-w \in \mathbb{Z}_{<0} .\end{cases}
\end{aligned}
$$

Using the symmetry in Remark 3.3(ii), we write $P^{+}(y, p, e)=(-q)^{y-p} P^{+}(p, e, y)$, $P^{+}(y, a, c-l-e-w)=(-q)^{l+e+w+y-c} P^{+}(c-l-e-w, a, y)$ and $P^{+}(c-l-e-$ $w, c, e)=P^{+}(c-l-e-w, e, c)$. Next use the explicit expressions of $P^{+}$and $P^{0}$ in terms of Wall polynomials and $q$-Bessel functions to find

$$
\begin{aligned}
& \sum_{p=0}^{\infty} q^{2 p} q^{p(a-c+e-y)}\left(q^{2+2 p} ; q^{2}\right)_{\infty} p_{c}\left(q^{2 p} ; q^{2 a-2 c} ; q^{2}\right) \\
& \quad \times p_{y}\left(q^{2 p} ; q^{2 e-2 y} ; q^{2}\right) J_{a-c+e-y}\left(q^{p-l-y-e-w} ; q^{2}\right) \\
& =C(-q)^{y+c}\left(q^{2+2(c-l-e-w)} ; q^{2}\right)_{\infty} \\
& \quad \times p_{c}\left(q^{2(c-l-e-w)} ; q^{2 e-2 c} ; q^{2}\right) p_{y}\left(q^{2(c-l-e-w)} ; q^{2 a-2 y} ; q^{2}\right),
\end{aligned}
$$

where

$$
C=q^{c(a-c)} q^{y(e-y)} q^{(-l-e-w)(e-c)} q^{(c-l-e-w-y)(a-y)} \frac{\left(q^{2+2 e-2 c}, q^{2+2 a-2 y} ; q^{2}\right)_{\infty}}{\left(q^{2+2 a-2 c}, q^{2+2 e-2 y} ; q^{2}\right)_{\infty}} .
$$

Replacing $(c, a-c, y, e-y,-l-y-e-w)$ by $\left(n, \sigma, m, \nu-\sigma, z^{\prime}\right)$ we obtain the result for $z=q^{z^{\prime}}, z^{\prime} \in \mathbb{Z}, \nu, \sigma \in \mathbb{Z}$ after a straightforward calculation.

Replacing $q^{z^{\prime}}$ by $z$, and removing $z^{\nu}$ from both sides, we see that we can extend by analytic continuation in $z$. Similarly, we can extend analytically in $\nu$ to $\Re \nu>-1$. Since the resulting identity is a Laurent polynomial in $q^{2 \sigma}$ and the convergence is uniform in $q^{2 \sigma}$ on bounded sets we find that the resulting identity is valid for arbitrary $\sigma \in \mathbb{C}$.

\section{NoTE ADDED IN PROOF}

In J. Math. Phys. 55 (2014), 101702, Groenevelt provides an alternative proof to Theorem 5.1 by studying Racah coefficients for the quantized function algebra of the quantum $S U(2)$ group. Moreover, Groenevelt presents generalizations of the result as well.

\section{ACKNOWLEDGEMENT}

The authors thank the referees for useful comments, which have improved the readability of the paper. 


\section{REFERENCES}

[1] Kenny De Commer, On a correspondence between $\mathrm{SU}_{q}(2), \widetilde{E}_{q}(2)$ and $\widetilde{\mathrm{SU}}_{q}(1,1)$, Comm. Math. Phys. 304 (2011), no. 1, 187-228, DOI 10.1007/s00220-011-1208-y. MR2793934 (2012c:16111)

[2] Kenny De Commer, On a Morita equivalence between the duals of quantum SU(2) and quantum $\tilde{E}(2)$, Adv. Math. 229 (2012), no. 2, 1047-1079, DOI 10.1016/j.aim.2011.10.011. MR.2855086

[3] A. Erdélyi, The Hankel Transform of a Product of Whittaker's Functions, J. London Math. Soc. S1-13, no. 2, 146, DOI 10.1112/jlms/s1-13.2.146. MR.1574143

[4] George Gasper and Mizan Rahman, Basic hypergeometric series, 2nd ed., Encyclopedia of Mathematics and its Applications, vol. 96, Cambridge University Press, Cambridge, 2004. With a foreword by Richard Askey. MR2128719 (2006d:33028)

[5] Mourad E. H. Ismail, Classical and quantum orthogonal polynomials in one variable, Encyclopedia of Mathematics and its Applications, vol. 98, Cambridge University Press, Cambridge, 2009. With two chapters by Walter Van Assche; With a foreword by Richard A. Askey; Reprint of the 2005 original. MR2542683 (2010i:33001)

[6] R. Koekoek, R.F. Swarttouw, The Askey-scheme of hypergeometric orthogonal polynomials and its q-analogue, online at http://aw.twi.tudelft.nl/ ${ }^{\sim}$ koekoek/askey.html, Report 9817, Technical University Delft, 1998.

[7] H. T. Koelink, The quantum group of plane motions and the Hahn-Exton q-Bessel function, Duke Math. J. 76 (1994), no. 2, 483-508, DOI 10.1215/S0012-7094-94-07617-5. MR.1302322 (96a:33023)

[8] Erik Koelink, Addition formulas for $q$-special functions, Special functions, $q$-series and related topics (Toronto, ON, 1995), Fields Inst. Commun., vol. 14, Amer. Math. Soc., Providence, RI, 1997, pp. 109-129. MR1448684 (98d:33013)

[9] Erik Koelink and Jasper V. Stokman, The Askey-Wilson function transform scheme, Special functions 2000: current perspective and future directions (Tempe, AZ), NATO Sci. Ser. II Math. Phys. Chem., vol. 30, Kluwer Acad. Publ., Dordrecht, 2001, pp. 221-241, DOI 10.1007/978-94-010-0818-1_9. MR2006290 (2005b:33019)

[10] K. S. Kölbig and H. Scherb, On a Hankel transform integral containing an exponential function and two Laguerre polynomials, J. Comput. Appl. Math. 71 (1996), no. 2, 357-363, DOI 10.1016/0377-0427(95)00224-3. MR1399902 (97c:65011)

[11] Tom H. Koornwinder, The addition formula for little $q$-Legendre polynomials and the SU(2) quantum group, SIAM J. Math. Anal. 22 (1991), no. 1, 295-301, DOI 10.1137/0522018. MR:1080160 (92f:33025)

[12] Tom H. Koornwinder, Askey-Wilson polynomials as zonal spherical functions on the $\mathrm{SU}(2)$ quantum group, SIAM J. Math. Anal. 24 (1993), no. 3, 795-813, DOI 10.1137/0524049. MR $1215439(94 \mathrm{k}: 33042)$

[13] Tom H. Koornwinder and René F. Swarttouw, On q-analogues of the Fourier and Hankel transforms, Trans. Amer. Math. Soc. 333 (1992), no. 1, 445-461, DOI 10.2307/2154118. MR.1069750 (92k:33013)

[14] E. Christopher Lance, An explicit description of the fundamental unitary for $\mathrm{SU}(2)_{q}$, Comm. Math. Phys. 164 (1994), no. 1, 1-15. MR1288151 (95h:46116)

[15] P. Podleś, Quantum spheres, Lett. Math. Phys. 14 (1987), no. 3, 193-202, DOI 10.1007/BF00416848. MR919322 (89b:46081)

[16] Mizan Rahman, A simple proof of Koornwinder's addition formula for the little q-Legendre polynomials, Proc. Amer. Math. Soc. 107 (1989), no. 2, 373-381, DOI 10.2307/2047827. MR.979214 (91a:33013)

[17] Thomas Timmermann, An invitation to quantum groups and duality, EMS Textbooks in Mathematics, European Mathematical Society (EMS), Zürich, 2008. From Hopf algebras to multiplicative unitaries and beyond. MR2397671 (2009f:46079)

[18] S. L. Woronowicz, Twisted SU(2) group. An example of a noncommutative differential calculus, Publ. Res. Inst. Math. Sci. 23 (1987), no. 1, 117-181, DOI 10.2977/prims/1195176848. MR890482 (88h:46130)

[19] S. L. Woronowicz, Quantum E(2) group and its Pontryagin dual, Lett. Math. Phys. 23 (1991), no. 4, 251-263, DOI 10.1007/BF00398822. MR1152695(93b:17058) 
[20] S. L. Woronowicz, Quantum SU(2) and E(2) groups. Contraction procedure, Comm. Math. Phys. 149 (1992), no. 3, 637-652. MR1186047 (93k:58015)

Department of Mathematics, University of Cergy-Pontoise, UMR CNRS 8088, F95000 Cergy-Pontoise, France

Current address: Department of Mathematics, Vrije Universiteit Brussel, VUB, B-1050 Brussels, Belgium

E-mail address: Kenny.De.Commer@vub.ac.be

Radboud Universiteit Nijmegen, IMAPP, FNWI, HeyendaAlseweg 135, 6525 AJ NijMEGEN, THE NETHERLANDS

E-mail address: e.koelink@math.ru.nl 